OPEN ACCESS

Edited by:

Gianluca laniro,

Agostino Gemelli University

Polyclinic, Italy

Reviewed by:

Christopher Staley,

University of Minnesota Twin Cities,

United States

Giusi Desirè Sciumè,

University of Pisa, Italy

${ }^{*}$ Correspondence:

Chunhui Li

lichunhui@csu.edu.cn

Anhua Wu

xywuanhua@csu.edu.cn

Specialty section:

This article was submitted to Microbiome in Health and Disease,

a section of the journal

Frontiers in Cellular and Infection

Microbiology

Received: 13 January 2020 Accepted: 09 April 2020

Published: 11 May 2020

Citation:

Duan J, Meng X, Liu S, Zhou P, Zeng C, Fu C, Dou Q, WU A and Li C (2020) Gut Microbiota Composition

Associated With Clostridium

difficile-Positive Diarrhea and

C. difficile Type in ICU Patients.

Front. Cell. Infect. Microbiol. 10:190.

doi: 10.3389/fcimb.2020.00190

\section{Gut Microbiota Composition Associated With Clostridium difficile-Positive Diarrhea and C. difficile Type in ICU Patients}

\author{
Juping Duan ${ }^{1,2}$, Xiujuan Meng ${ }^{1}$, Sidi Liu ${ }^{1}$, Pengcheng Zhou ${ }^{1}$, Cui Zeng ${ }^{1}$, Chenchao Fu ${ }^{1}$, \\ Qingya Dou ${ }^{1}$, Anhua $W^{1 *}$ and Chunhui $\mathrm{Li}^{1 *}$ \\ ${ }^{1}$ Infection Control Center, Xiangya Hospital, Central South University, Changsha, China, ${ }^{2}$ Changsha Hospital of Traditional \\ Chinese Medicine, Changsha, China
}

The gut microbiota composition of intensive care unit (ICU) patients suffering from Clostridium difficile-positive diarrhea $(\mathrm{CDpD})$ is poorly understood. This prospective study aims to use 16S rDNA (and metagenome) sequencing to compare the microbiota composition of 58 (and 5) ICU patients with CDpD (CDpD group), 33 (and 4) ICU patients with $C$. difficile-negative diarrhea (CDnD group), and 21 (and 5) healthy control subjects (control group), as well as $C D p D$ patients in the $A^{+} B^{+}(N=34 ; A / B$ : C. difficile $T c d A / B)$, $\mathrm{A}^{-} \mathrm{B}^{+}(N=7)$, and $\mathrm{A}^{-} \mathrm{B}^{-}(N=17)$ subgroups. For $16 \mathrm{~S}$ rDNA data, OTU clustering (tool: UPARSE), taxonomic assignment (tool: RDP classifier), $\alpha$-diversity, and $\beta$-diversity analyses (tool: QIIME) were conducted. For metagenome data, metagenome assembly (tool: SOAPdenovo), gene calling (tools: MetaGeneMark, CD-HIT, and SoapAligner), unigene alignment (tool: DIAMOND), taxon difference analysis (tool: Metastats), and gene annotation (tool: DIAMOND) were performed. The microbial diversity of the CDpD group was lower than that of the $\mathrm{CDnD}$ and control groups. The abundances of 10 taxa (e.g., Deferribacteres, Cryptomycota, Acetothermia) were significantly higher in the CDpD group than in the CDnD group. The abundances of Saccharomycetes and Clostridia were significantly lower in $\mathrm{CDpD}$ in comparison with control. Some taxa were significantly different between the $\mathrm{A}^{+} \mathrm{B}^{+}$and $\mathrm{A}^{-} \mathrm{B}^{-}$subgroups. $\mathrm{CDpD}$ might relate to a decrease in beneficial taxa (i.e., Saccharomycetes and Clostridia) and an increase in harmful taxa (e.g., Deferribacteres, Cryptomycota, Acetothermia) in gut microbiota of ICU patients. C. difficile toxin type might be slightly associated with gut microbiota composition.

\footnotetext{
Keywords: 16S rDNA sequencing, Clostridium difficile-associated diarrhea, gut microbiota, metagenome sequencing, $T c d A / T c d B$, Clostridium difficile infection, $C D I$, Clostridium difficile-positive diarrhea
}

\section{INTRODUCTION}

Antibiotics are often used in treating intensive care unit (ICU) patients (Vincent et al., 2016). However, antibiotic treatment is the most crucial risk factor associated Clostridium difficile (also known as Peptoclostridium difficile) infection (CDI) (Stevens et al., 2011), as antibiotics adversely affect the indigenous gut microbiota composition and decrease colonization resistance to C. difficile (Britton and Young, 2012). The clinical symptoms of CDI range from mild diarrhea to severe 
complications such as pseudomembranous colitis, toxic megacolon, bowel perforation, and death (Yassin et al., 2001; Rupnik et al., 2009; Shivashankar et al., 2013; Leffler and Lamont, 2015). The occurrence of CDI is $7.4 \sim 14.1$ cases per 10,000 patient-days in ICU (Centers for Disease Control Prevention (CDC), 2012; Lee et al., 2015; Li et al., 2018).

Nevertheless, current antibiotic therapies for CDI such as vancomycin have limited efficacy (Bagdasarian et al., 2015), and fecal microbiota transplantation (FMT) is recommended as an alternative therapy in treating highly recurrent CDI that has failed to respond to vancomycin treatment (Kelly et al., 2015). The success of FMT emphasizes the importance of restoring the gut microbiome in CDI patients.

In recent years, high-throughput deep-sequencing of $16 \mathrm{~S}$ rDNA and the metagenome has been applied to investigating microbiota composition. Milani et al. used $16 \mathrm{~S}$ rDNA and metagenome sequencing to study the gut microbiota compositions of three groups of elderly (age $\geq 65$ ) hospitalized patients, involving $30 \mathrm{CDI}$-negative patients not exposed to antibiotics, 29 CDI-negative patients exposed to antibiotics, and 25 CDI-positive patients (Milani et al., 2016). The microbial diversity of the CDI-positive group was significantly lower than that of the CDI-negative group. CDI was associated with the decrease in gut commensal bacteria such as Bacteroides, Alistipes, Lachnospira, and Barnesiella, while antibiotic treatment in CDI-negative patients might lead to the depletion of commensal bacteria such as Alistipes (Milani et al., 2016). Schubert et al. utilized $16 \mathrm{~S}$ rDNA sequencing to study the gut microbiota compositions of CDI cases, diarrheal controls, and nondiarrheal controls (Schubert et al., 2014). Statistical models were developed for CDI and diarrhea by incorporating clinical and demographic data with microbiome data, and loss of several species in Ruminococcaceae, Lachnospiraceae, Bacteroides and Porphyromonadaceae might be associate with CDI (Schubert et al., 2014). However, the microbiome alterations related with diarrhea and $C$. difficile-positive diarrhea $(\mathrm{CDpD})$ have not been completely elucidated in ICU patients. Generally, only $C$. difficile that produces toxin $\mathrm{A}$ (an intestinotoxin, $T c d A$ ) and/or toxin B (a cytotoxin, $T c d B$ ) can cause gastrointestinal diseases in humans. However, relationships between $C$. difficile type (i.e., $\mathrm{A}^{+} \mathrm{B}^{+}, \mathrm{A}^{-} \mathrm{B}^{+}$, and $\mathrm{A}^{-} \mathrm{B}^{-}$) and the gut microbiota composition have not been investigated.

In this prospective study, we utilized $16 \mathrm{~S}$ rDNA and metagenome deep-sequencing to characterize the gut microbiota composition in healthy subjects, $\mathrm{CDpD}$ ICU patients, and $C$. difficile negative diarrhea $(\mathrm{CDnD})$ ICU patients, as well as the gut microbiota composition in ICU patients with $\mathrm{A}^{+} \mathrm{B}^{+}$ $\mathrm{CDpD}, \mathrm{A}^{-} \mathrm{B}^{+} \mathrm{CDpD}$, and $\mathrm{A}^{-} \mathrm{B}^{-} \mathrm{CDpD}$. Our results may help to elucidate the mechanisms underlying diarrhea and $\mathrm{CDpD}$ development in ICU patients and identify potential candidates for curative or preventive microbe therapy.

Abbreviations: CDI, C. difficile infection; $\mathrm{CDpD}$, C. difficile-positive diarrhea; FMT, Fecal microbiota transplantation; ICU, Intensive care unit; OTU, Operational taxonomic unit.

\section{MATERIALS AND METHODS}

\section{Study Population and Sample Collection}

Xiangya Hospital is a 3,500-bed urban tertiary teaching hospital in Changsha, Hunan Province, China, and admits $~ 90,000$ patients per annum. The hospital has a General ICU (35 beds). In this study, a total of 112 subjects were enrolled between March 2014 and December 2014. Subjects included 21 nonpregnant healthy individuals (to whom antibiotics had not been administered within 1 month and without any signs of diarrhea within 7 days before sample collection) and 91 non-pregnant ICU patients with signs of hospital-onset diarrhea (where diarrhea occurred $48 \mathrm{~h}$ after hospital admission with stool three or more times within $24 \mathrm{~h}$ ). Subjects with inflammatory bowel disease and diarrhea that occurred less than $48 \mathrm{~h}$ after hospital admission were excluded from the study. For the ICU patients with hospitalonset diarrhea, C. difficile was screened within $24 \mathrm{~h}$ after sampling (at least $300-\mu \mathrm{l}$ diarrheal stool samples frozen at $-20^{\circ} \mathrm{C}$ for PCR) using 16S rDNA-PCR (PS13: 5'-GGAGGCAGCAGTGGGGAA TA-3', PS14: 5'-TGACGGGCGGTGTGTACAAG-3') (Persson et al., 2011), sequencing, and BLAST alignment. Patients with positive results were classified into the $\mathrm{CDpD}$ group $(N=58)$, and patients with negative results were classified into the $\mathrm{CDnD}$ group $(N=33)$.

Diarrheal stool samples (at least $300 \mu \mathrm{l}$ ) obtained from patients and non-diarrheal stool samples (at least $300 \mu \mathrm{l}$ ) obtained from healthy individuals were frozen at $-80^{\circ} \mathrm{C}$ before sequencing in 2015. Then, $16 \mathrm{~S}$ rDNA V4 sequencing-based microbiota analysis was performed for stool samples in the $\mathrm{CDpD}, \mathrm{CDnD}$, and healthy control groups (CDpD-CDnDcontrol 16S rDNA V4 sequencing analysis). Moreover, 14 samples were selected from these groups (five samples in the $\mathrm{CDpD}$ group, four samples in the $\mathrm{CDnD}$ group, and five samples in the control group) to conduct genome sequencing-based metagenomics analysis (CDpD-CDnD-control metagenome sequencing analysis).

According to the PCR results of $C$. difficile $T c d A$ (gene for toxin A; Forward primer: 5'-AGATTCCTATATTTACATGAC AATAT-3', Reverse primer: 5' -GTATCAGGCATAAAGTAATAT ACTTT-3') (Lemee et al., 2004) and TcdB (gene for toxin B; NK104: 5'-GTGTAGCAATGAAAGTCCAAGTTTACGC-3', NK105: 5'-CACTTAGCTCTTTGATTGCTGCACCT-3') (Kato et al., 1998), patients in the CDpD group were classified into the $\mathrm{A}^{+} \mathrm{B}^{+}$subgroup $(N=34), \mathrm{A}^{-} \mathrm{B}^{+}$subgroup $(N=7)$, and $\mathrm{A}^{-} \mathrm{B}^{-}$subgroup $(N=17)$, and $16 \mathrm{~S} \mathrm{rDNA}$ V4 sequencing-based microbiota analysis was then performed for stool specimens in the $\mathrm{A}^{+} \mathrm{B}^{+}, \mathrm{A}^{-} \mathrm{B}^{+}$, and $\mathrm{A}^{-} \mathrm{B}^{-}$subgroups (CDpD subgroups $16 \mathrm{~S}$ rDNA V4 sequencing analysis).

\section{Clinical Data Collection}

For each subject, clinical data were collected, including age, gender, duration of hospital stay, C. difficile detection, other diseases (i.e., diabetes, cancer, hematopathy, respiratory failure, renal insufficiency, and tuberculosis), surgery, and exposure to immunosuppressants, glucocorticoids, and antibiotics. 


\section{Amplification and Sequencing of 16S rDNA}

As previously described (Schubert et al., 2014; Milani et al., 2016), DNA was extracted from stool samples, and the V4region (primers: $515 \mathrm{~F}$ and $806 \mathrm{R}$ ) of $16 \mathrm{~S}$ rDNA were amplified and sequenced using an Illumina HiSeq2500 PE250 Pairedend sequencer. As detailed in the Supplemental Materials, $16 \mathrm{~S}$ rDNA-based microbiota analysis was performed, including chimera sequence removal, operational taxonomic unit (OTU) clustering, taxonomic assignment, phylogenetic relationship, $\alpha-$ diversity analysis, and $\beta$-diversity analysis [unweighted unifrac distance, weighted unifrac distance, sample clustering tree, and principal coordinate analysis (PCoA)].

\section{Genome Sequencing}

Total metagenomic DNA was isolated from each stool sample (Milani et al., 2016). After obtaining fragments of $300 \mathrm{bp}$ (ultrasonic method), end repairing, adding poly-A, adding sequencing adapters, purification, and PCR, DNA libraries were obtained and then sequenced using an Illumina HiSeq2500 Paired-end sequencer with a $2 \times 150$ bp read length. Metagenomic data were analyzed (Supplemental Materials), including metagenome assembly de novo, gene calling, alignment of unigenes to reference genomes (microbial reference genomes of bacteria, fungi, archaea, and viruses in the NR database of the National Center for Biological Information), taxonomical analysis and pathway annotation.

\section{Statistical Analyses}

Statistical analyses were performed using SPSS 19.0 software to determine the differences in clinical characteristics among groups (i.e., $\mathrm{CDpD}, \mathrm{CDnD}$, and control groups), as well as subgroups (i.e., $\mathrm{A}^{+} \mathrm{B}^{+}, \mathrm{A}^{-} \mathrm{B}^{+}$, and $\mathrm{A}^{-} \mathrm{B}^{-}$subgroups). For continuous variables, comparison among three groups was performed using one-way ANOVA, whereas comparison between two groups was conducted using independent-samples t-test. In oneway ANOVA, least-significant-difference test and Dunnett's T3 method were used under the conditions of equal variance and unequal variance, respectively. $P<0.05$ was set as the criterion for statistical difference. For categorical variables, Fisher's exact test and Pearson's chi-square test were performed.

\section{RESULTS}

\section{Clinical Features of Subjects}

For CDpD-CDnD-control 16S rDNA V4 sequencing analysis, subjects in the CDpD group $(N=58), \mathrm{CDnD}$ group $(N=33)$, and control group $(N=21)$ were significantly different for age, but similar for gender. Subjects in the $\mathrm{CDpD}$ and $\mathrm{CDnD}$ groups were significantly different for antibiotics usage (i.e., exposure to antibiotics within 1 month before diarrhea) but similar for the other clinical features (Table $\mathbf{1}$ ).

For the $\mathrm{CDpD}$ subgroups $16 \mathrm{~S}$ rDNA V4 sequencing analysis, subjects in the $\mathrm{A}^{+} \mathrm{B}^{+}$subgroup $(N=34), \mathrm{A}^{-} \mathrm{B}^{+}$subgroup $(N$ $=7)$, and $\mathrm{A}^{-} \mathrm{B}^{-}$subgroup $(N=17)$ of the $\mathrm{CDpD}$ group were significantly different for antibiotics usage but similar for the other clinical features (Table 1).
For $\mathrm{CDpD}-\mathrm{CDnD}$-control metagenome sequencing analysis, subjects in the CDpD group $(N=5), \mathrm{CDnD}$ group $(N=4)$, and control group $(N=5)$ were significantly different for age but similar for gender. Subjects in the $\mathrm{CDpD}$ and $\mathrm{CDnD}$ groups were significantly different for antibiotics usage but similar for the other clinical features (Table 1).

\section{CDpD-CDnD-Control 16S rDNA V4 Sequencing Analysis}

In $\alpha$-diversity analysis, the Chaol index, Shannon index, and number of observed species were assessed. The Chaol index (i.e., community richness) in the $\mathrm{CDpD}$ group was lower than that in the CDnD and control groups. The Shannon index (i.e., community diversity) in the $\mathrm{CDpD}$ group was similar to that in the CDnD group but much lower than that in the control group. The number of observed species in the $\mathrm{CDpD}$ group was much lower than in the $\mathrm{CDnD}$ and control groups. A total of 2,125 $16 \mathrm{~S}$ rDNA-based OTUs were clustered, among which 513 OTUs were shared by the three groups.

In $\beta$-diversity analysis, the un-weighted unifrac distances were $0.282,0.617$, and 0.61 between $\mathrm{CDnD}$ and control, between $\mathrm{CDpD}$ and control, and between $\mathrm{CDpD}$ and $\mathrm{CDnD}$, respectively (Figure 1A). PCoA was conducted based on un-weighted unifrac distance, and $\mathrm{CDpD}$ samples could be clearly separated from $\mathrm{CDnD}$ samples and controls (Figure 1B). Besides, the CDpD, $\mathrm{CDnD}$, and control groups could be distinguished from each other based on unweighted/weighted unifrac distance and relative abundance at the Phylum level (Figures 1C,D).

Relative abundances of the top 10 taxa in terms of Phylum, Class, Order, Family, and Genus were studied. At the Phylum level, the relative abundance of Firmicutes was lower whereas those of Proteobacteria and Verrucomicrobia were higher in the $\mathrm{CDpD}$ group when compared with the $\mathrm{CDnD}$ and control groups (Figures 1C,D). At the Class level, the relative abundances of Verrucomicrobiae and Gammaproteobacteria were higher in the $\mathrm{CDpD}$ group (Figure 2A). At the Order level, the relative abundances of Verrucomicrobiales and Enterobacteriales were higher in the $\mathrm{CDpD}$ group (Figure 2B). At the Family level, the relative abundances of Porphyromonadaceae, Verrucomicrobiaceae, and Enterobacteriaceae were higher in the CDpD group (Figure 2C). At the Genus level, the relative abundances of Parabacteroides and Akkermansia were higher in the CDpD group (Figure 2D).

The phylogenetic relationships between the representative sequences of all OTUs corresponding to the top 10 Genera are shown in Figure 2E. Additionally, a taxonomic tree was constructed (Figure 2F). The abundances of Porphyromonadaceae, Parabacteroides, Parabacteroides distasonis, and Bacteroides caccae in the $\mathrm{CDpD}$ group were greater than those in the $\mathrm{CDnD}$ and control groups. These taxa might be associated with $\mathrm{CDpD}$. Firmicutes, Clostridia, Clostridiales, Ruminococcaceae, Ruminococcus, Ruminococcus Bromii, and Ruminococcus callidus as well as Veillonellaceae and Megamonas were more abundant in the control group than in the $\mathrm{CDnD}$ and $\mathrm{CDpD}$ groups. These taxa might be associated with diarrhea. 
TABLE 1 | Demographic and clinical characteristics of the population in each experimental group.

\begin{tabular}{|c|c|c|c|c|c|c|c|c|c|c|c|c|c|}
\hline & & \multicolumn{4}{|c|}{$\begin{array}{l}\text { CDpD-CDnD-control 16S rDNA V4 } \\
\text { sequencing analysis }\end{array}$} & \multicolumn{4}{|c|}{$\begin{array}{l}\text { CDpD subgroups } 16 \mathrm{~S} \text { rDNA V4 } \\
\text { sequencing analysis }\end{array}$} & \multicolumn{4}{|c|}{$\begin{array}{l}\text { CDpD-CDnD-control genome } \\
\text { sequencing analysis }\end{array}$} \\
\hline & & $\begin{array}{l}\text { CDpD } \\
(N=58)\end{array}$ & $\begin{array}{l}\text { CDnD } \\
(N=33)\end{array}$ & $\begin{array}{l}\text { Control } \\
(N=21)\end{array}$ & $P$ & $\begin{array}{c}A^{+} B^{+} \\
(N=34)\end{array}$ & $\begin{array}{l}A^{-} B^{+} \\
(N=7)\end{array}$ & $\begin{array}{c}A^{-} B^{-} \\
(N=17)\end{array}$ & $P$ & $\begin{array}{l}\text { CDpD } \\
(N=5)\end{array}$ & $\begin{array}{l}\text { CDnD } \\
(N=4)\end{array}$ & $\begin{array}{l}\text { Control } \\
(N=5)\end{array}$ & $P$ \\
\hline \multirow[t]{4}{*}{ Age } & Mean (SD) & $55.3(15.9)$ & $58.8(15.6)$ & $36.6(9.5)$ & $<0.001$ & $56.8(17.3)$ & $54.4(10.1)$ & $52.6(15.3)$ & 0.684 & $54.2(21.4)$ & $72.0(11.7)$ & $38.8(13)$ & 0.035 \\
\hline & & \multicolumn{3}{|c|}{ CDpD vs. CDnD } & 0.276 & \multicolumn{3}{|c|}{$\mathrm{A}^{+} \mathrm{B}^{+}$vs. $\mathrm{A}^{-} \mathrm{B}+$} & 0.728 & \multicolumn{3}{|c|}{ CDpD vs. CDnD } & 0.132 \\
\hline & & \multicolumn{3}{|c|}{ CDpD vs. Control } & $<0.001$ & \multicolumn{3}{|c|}{$\mathrm{A}^{+} \mathrm{B}^{+}$vs. $\mathrm{A}^{-} \mathrm{B}^{-}$} & 0.392 & \multicolumn{3}{|c|}{ CDpD vs. Control } & 0.164 \\
\hline & & \multicolumn{3}{|c|}{ CDnD vs. Control } & $<0.001$ & \multicolumn{3}{|c|}{$A^{-} B^{+}$vs. $A^{-} B^{-}$} & 0.806 & \multicolumn{3}{|c|}{ CDnD vs. Control } & 0.011 \\
\hline \multirow[t]{2}{*}{ Gender } & Female & $14(24.1 \%)$ & $14(42.4 \%)$ & 8 (38.1\%) & 0.162 & 10 (29.4\%) & $0(0 \%)$ & $4(23.5 \%)$ & 0.253 & $3(60 \%)$ & 2 (50\%) & $0(0 \%)$ & 0.151 \\
\hline & Male & 44 (75.9\%) & $19(57.6 \%)$ & 13 (61.9\%) & & $24(70.6 \%)$ & 7 (100\%) & $13(76.5 \%)$ & & $2(40 \%)$ & $2(50 \%)$ & 5 (100\%) & \\
\hline \multirow[t]{4}{*}{ Inpatient days } & Mean (SD) & 29.4 (18.9) & $27.2(17.2)$ & NA & 0.577 & $31.1(20.4)$ & $27.4(11.4)$ & $26.9(18.6)$ & 0.738 & $23.4(19.8)$ & $39.3(7.7)$ & NA & 0.157 \\
\hline & & & & & & \multicolumn{3}{|c|}{$\mathrm{A}^{+} \mathrm{B}^{+}$vs. $\mathrm{A}^{-} \mathrm{B}^{+}$} & 0.649 & & & & \\
\hline & & & & & & \multicolumn{3}{|c|}{$A^{+} B^{+}$vs. $A^{-} B^{-}$} & 0.471 & & & & \\
\hline & & & & & & \multicolumn{3}{|c|}{$A^{-} B^{+}$vs. $A^{-} B^{-}$} & 0.955 & & & & \\
\hline With diabetes & Yes & $9(15.5 \%)$ & 2 (6.06\%) & NA & 0.183 & $6(17.6 \%)$ & $0(0 \%)$ & $3(17.6 \%)$ & 0.481 & $0(0 \%)$ & $0(0 \%)$ & NA & NA \\
\hline With cancer & Yes & $6(10.3 \%)$ & $4(12.1 \%)$ & NA & 0.794 & 3 (8.82\%) & $1(14.3 \%)$ & $2(11.8 \%)$ & 0.844 & $0(0 \%)$ & $1(25 \%)$ & NA & 0.444 \\
\hline With hematopathy & Yes & $2(3.45 \%)$ & $0(0 \%)$ & NA & 0.533 & 2 (5.88\%) & $0(0 \%)$ & $0(0 \%)$ & 0.650 & $0(0 \%)$ & $0(0 \%)$ & NA & NA \\
\hline With respiration failure & Yes & $7(12.1 \%)$ & $4(12.1 \%)$ & NA & 0.994 & $4(11.8 \%)$ & $2(28.6 \%)$ & $1(5.88 \%)$ & 0.285 & $0(0 \%)$ & $1(25 \%)$ & NA & 0.444 \\
\hline With renal insufficiency & Yes & 5 (8.62\%) & 3 (9.09\%) & NA & 0.939 & $4(11.8 \%)$ & $1(14.3 \%)$ & $0(0 \%)$ & 0.295 & $1(20 \%)$ & $1(25 \%)$ & NA & 1.00 \\
\hline With tuberculosis & Yes & $0(0 \%)$ & 1 (3.03\%) & NA & 0.363 & $0(0 \%)$ & $0(0 \%)$ & $0(0 \%)$ & NA & $0(0 \%)$ & $0(0 \%)$ & NA & NA \\
\hline Antibiotics usage & Yes & $39(67.2 \%)$ & $8(24.2 \%)$ & NA & $<0.001$ & $29(85.3 \%)$ & $5(71.4 \%)$ & $5(29.4 \%)$ & $<0.001$ & $5(100 \%)$ & $1(25 \%)$ & NA & 0.048 \\
\hline Immunosuppressant usage & Yes & $0(0 \%)$ & $0(0 \%)$ & NA & NA & $0(0 \%)$ & $0(0 \%)$ & $0(0 \%)$ & NA & $0(0 \%)$ & $0(0 \%)$ & NA & NA \\
\hline Glucocorticoids usage & Yes & $5(8.62 \%)$ & $0(0 \%)$ & NA & 0.083 & 3 (8.82\%) & $2(28.6 \%)$ & $0(0 \%)$ & 0.085 & $0(0 \%)$ & $0(0 \%)$ & NA & NA \\
\hline Surgery before diarrheal & Yes & 27 (46.6\%) & 17 (51.5\%) & NA & 0.649 & $13(38.2 \%)$ & $4(57.1 \%)$ & $10(58.8 \%)$ & 0.318 & $5(100 \%)$ & $2(50 \%)$ & NA & 0.167 \\
\hline
\end{tabular}

$C D p D$, Clostridium difficile-positive diarrhea; CDnD, C. difficile-negative diarrhea; SD, standard deviation; NA, not applicable. Antibiotics usage, exposure to antibiotics within 1 month before diarrheal; Immunosuppressant usage, exposure to immunosuppressant within 1 month before diarrheal; Glucocorticoids usage, exposure to Glucocorticoids within 1 month before diarrheal; Surgery before diarrheal, acceptance of surgery before diarrheal. 
A

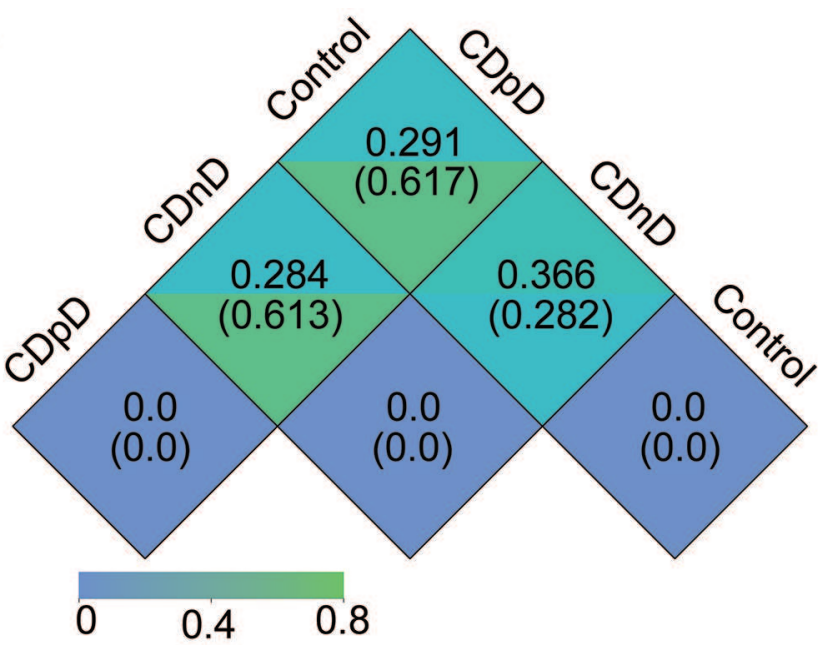

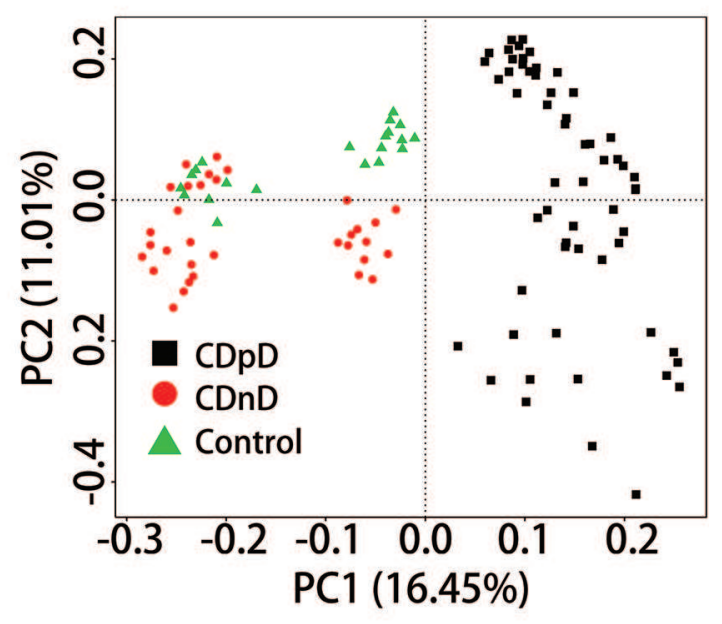

C

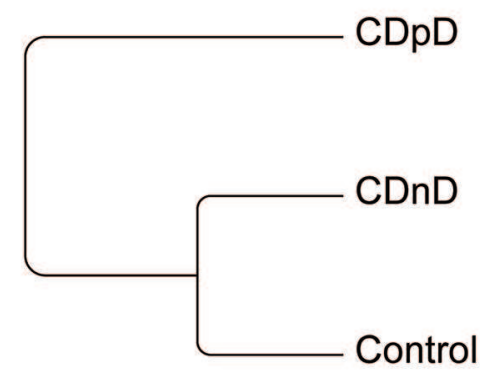

Unweighted Unifrac Distance

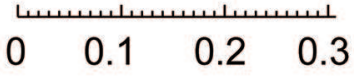

D

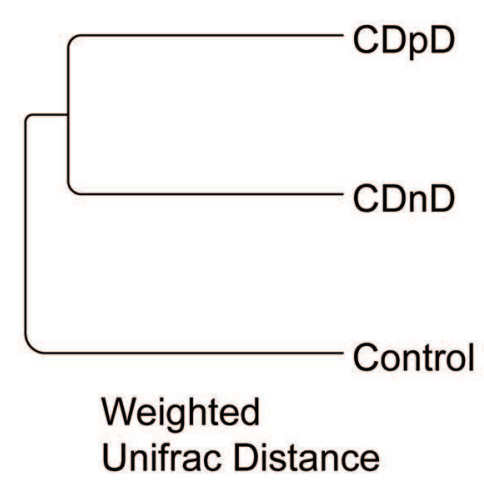

$\begin{array}{llll}0 & 0.05 & 0.1 & 0.15\end{array}$
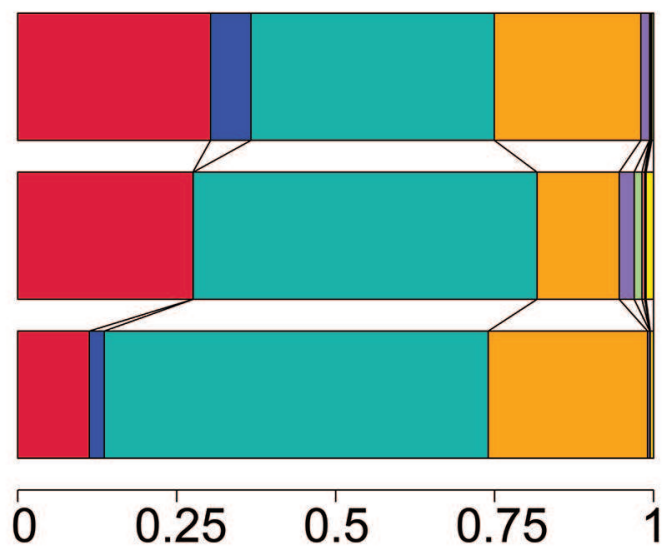

Relative Abundance at Phylum Level $\square$ Proteobacteria

$\square$ Verrucomicrobia

Firmicutes

Bacteroidetes

Actinobacteria

Spirochaetes

Cyanobacteria

Synergistetes

Fusobacteria

Euryarchaeota

Others
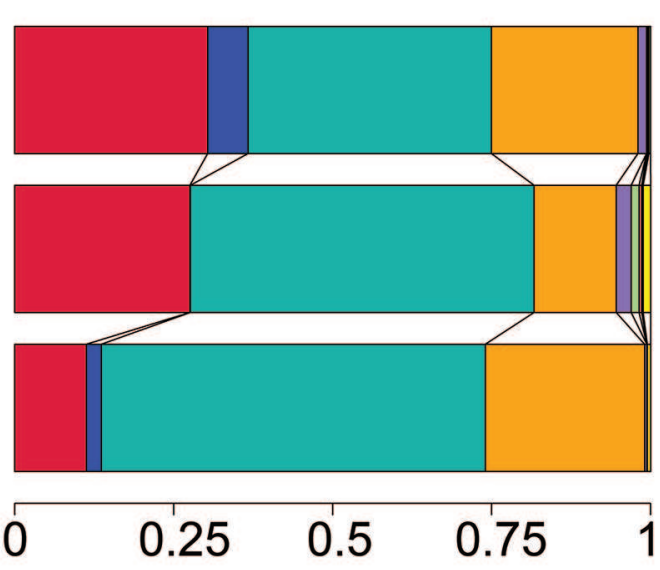

Relative Abundance at Phylum Level $\square$ Proteobacteria

Verrucomicrobia

Firmicutes

Bacteroidetes

Actinobacteria

Spirochaetes

Cyanobacteria

Synergistetes

Fusobacteria

Euryarchaeota

Others

FIGURE 1 | $\alpha$-diversity and $\beta$-diversity analysis in CDpD-CDnD-control $16 S$ rDNA V4 sequencing analysis. (A) Unifrac distance between groups. Upper number: weighted unifrac distance; lower number: un-weighted unifrac distance. Both numbers represent the index for differences in taxon-diversity between groups. (B) PCoA based on unweighted unifrac distance. (C) Sample clustering based on unweighted unifrac distance and relative abundance at Phylum level. (D) Sample clustering based on weighted unifrac distance and relative abundance at Phylum level. 


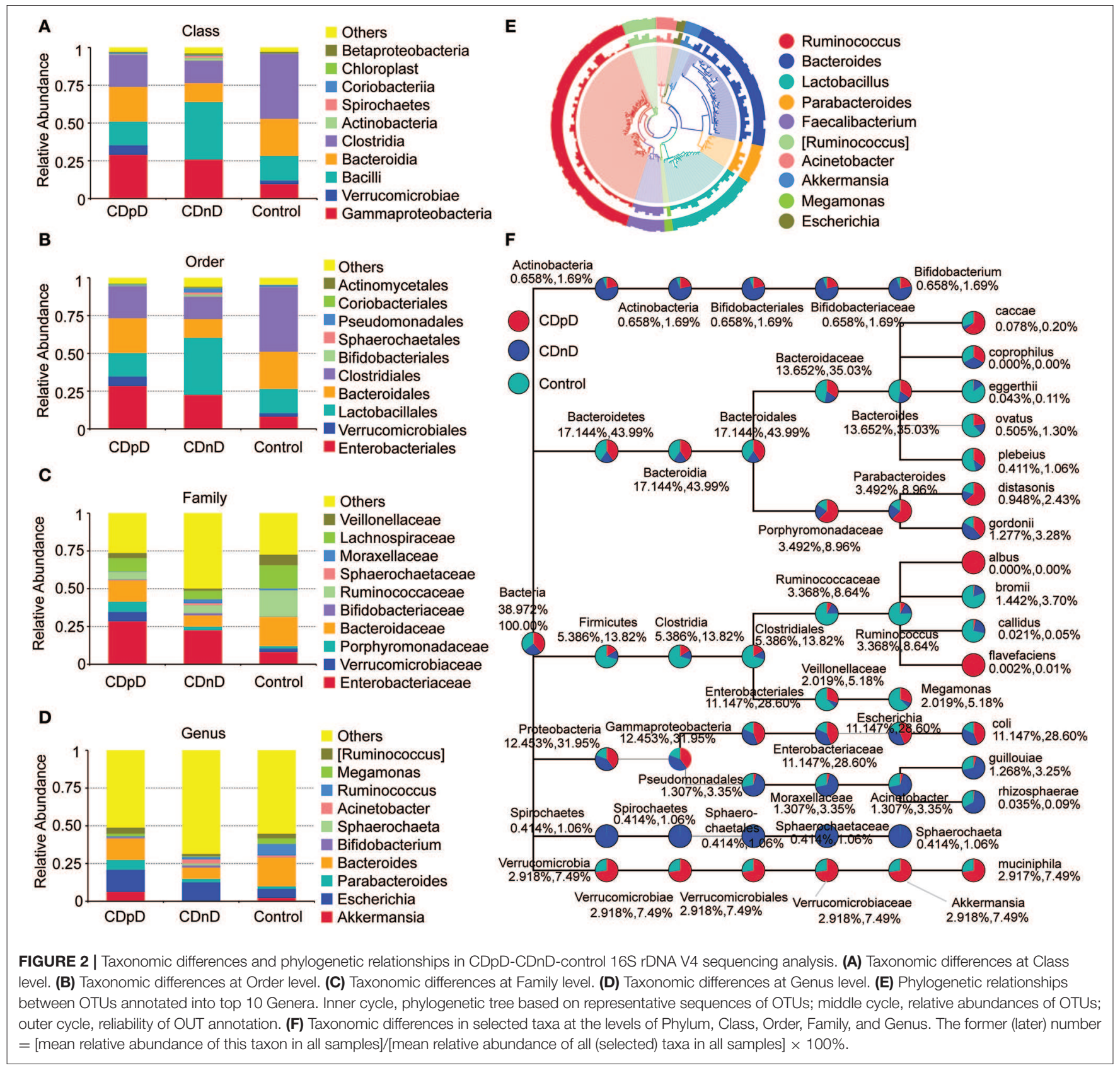

\section{CDpD-CDnD-Control Metagenome Sequencing Analysis}

In core-pan gene analysis, the number of non-redundant genes declined along with the increase in sample number in the coregene curve, whereas it increased along with the increase in sample number in the pan-gene curve. These results supported a further detailed analysis of metagenome sequencing data. A total of 591,552 genes were predicted based on metagenome sequences.

In $\mathrm{CDpD}$-CDnD-control metagenome sequencing analysis, significant differences were identified between the $\mathrm{CDpD}$ and $\mathrm{CDnD}$ groups in the abundances of 10 taxa (Dictyoglomi, Acetothermia, Cryptomycota, Chytridiomycota,
Glomeromycota, Planctomycetes, Aquificae, Deferribacteres, Poribacteria, and Armatimonadetes; Figures 3A,B). Also, significant differences were found between the $\mathrm{CDpD}$ and control groups in the abundances of Holophagae, Bacteroidia, Saccharomycetes, and Clostridia (Figures 3C,D). In both CDpD-CDnD-control 16S rDNA V4 sequencing analysis and $\mathrm{CDpD}$-CDnD-control metagenome sequencing analysis, the relative abundance of Clostridia in the $\mathrm{CDpD}$ group was lower than in the control group.

Based on the Kyoto Encyclopedia of Genes and Genomes (KEGG) database, most of the predicted genes were related with Metabolism (e.g., Carbohydrate metabolism, Amino 


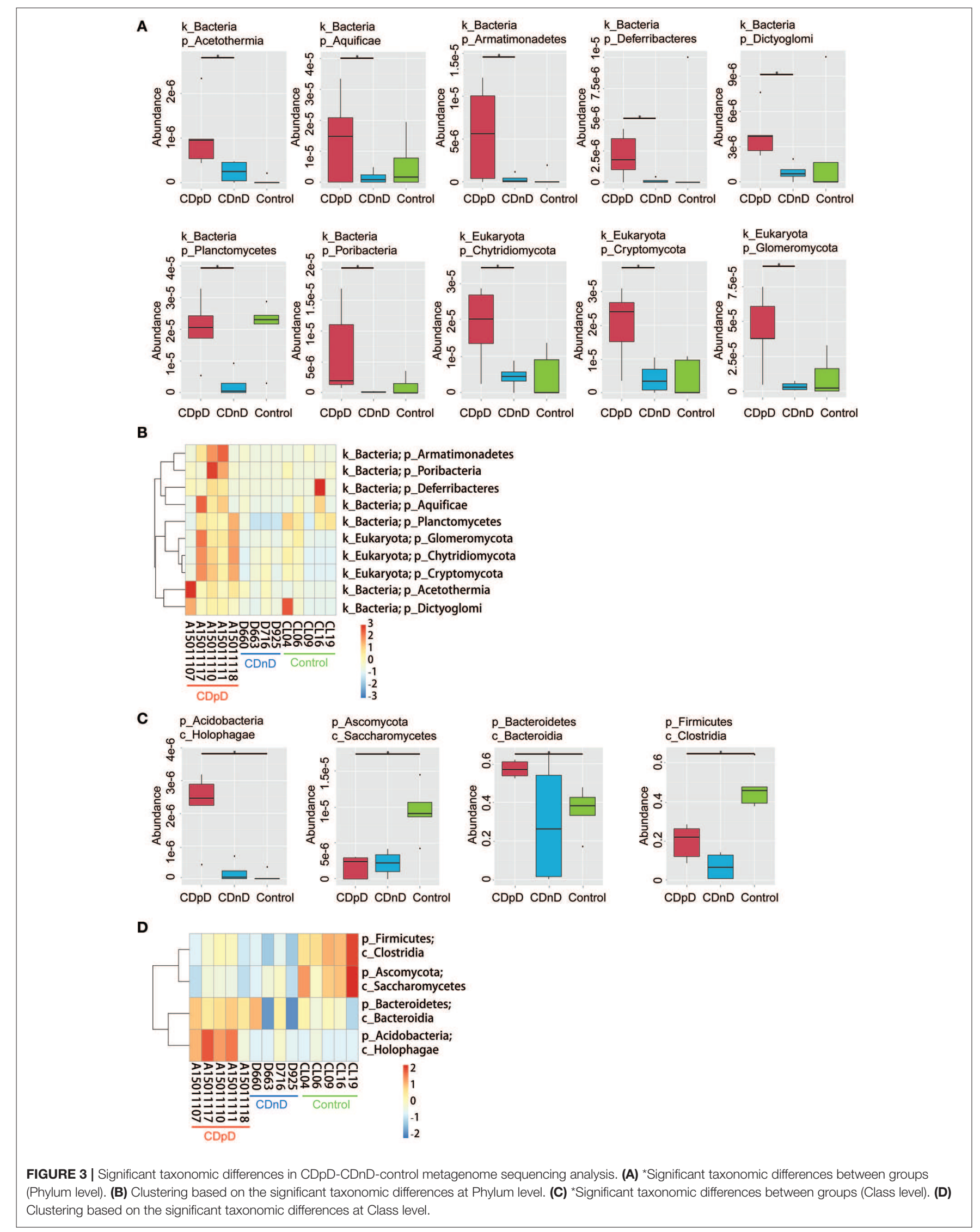




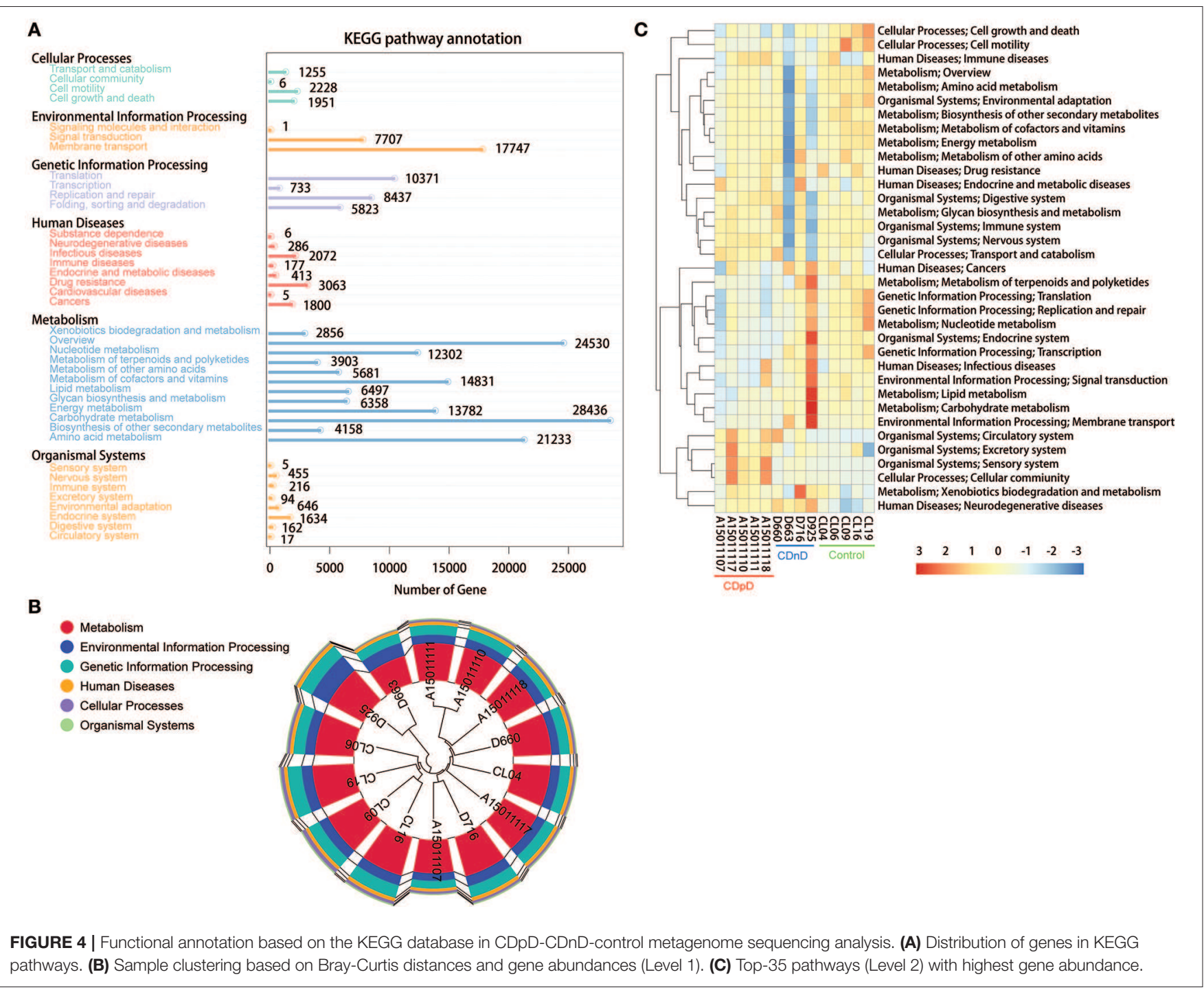

acid metabolism), Environmental Information Processing (e.g., Membrane transport), and Genetic Information Processing (e.g., Translation) (Figure 4A). Samples were clustered based on BrayCurtis distances and gene abundances (KEGG level 1), and control samples were closely clustered (Figure 4B). The top35 pathways with the highest gene abundance were identified (KEGG level 2, Figure 4C). Compared with CDnD and control samples, CDpD samples showed significantly higher abundances in pathways such as "Cellular community," "Excretory system," and "Circulatory system." Besides, "Lysine biosynthesis" and "Coenzyme B biosynthesis" were only annotated in CDpD samples when compared with controls.

\section{CDpD Subgroups 16S rDNA V4 Sequencing Analysis}

In $\alpha$-diversity analysis, the Chaol index, Shannon index, and number of observed species in the $\mathrm{A}^{+} \mathrm{B}^{+}$subgroup was much higher than in the $\mathrm{A}^{-} \mathrm{B}^{+}$and $\mathrm{A}^{-} \mathrm{B}^{-}$subgroups.

In $\beta$-diversity analysis, the unweighted unifrac distances were $0.471,0.429$, and 0.466 between $\mathrm{A}^{-} \mathrm{B}^{+}$and $\mathrm{A}^{-} \mathrm{B}^{-}$, between
$\mathrm{A}^{+} \mathrm{B}^{+}$and $\mathrm{A}^{-} \mathrm{B}^{-}$, and between $\mathrm{A}^{+} \mathrm{B}^{+}$and $\mathrm{A}^{-} \mathrm{B}^{+}$(Figure 5A). In $\beta$-diversity analysis, the weighted unifrac distances were 0.257 , 0.254 , and 0.252 between $\mathrm{A}^{-} \mathrm{B}^{+}$and $\mathrm{A}^{-} \mathrm{B}^{-}$, between $\mathrm{A}^{+} \mathrm{B}^{+}$and $\mathrm{A}^{-} \mathrm{B}^{-}$, and between $\mathrm{A}^{+} \mathrm{B}^{+}$and $\mathrm{A}^{-} \mathrm{B}^{+}$(Figure 5A). These results indicated that the differences in taxon diversity between these subgroups were similar.

In PCoA based on unweighted unifrac distance, high inter-sample variability was found across groups, and $\mathrm{A}^{+} \mathrm{B}^{+}$ samples could not be distinguished from $\mathrm{A}^{-} \mathrm{B}^{+}$or $\mathrm{A}^{-} \mathrm{B}^{-}$ samples (Figure 5B). However, the $\mathrm{A}^{+} \mathrm{B}^{+}, \mathrm{A}^{-} \mathrm{B}^{+}$, and $\mathrm{A}^{-} \mathrm{B}^{-}$ subgroups could be distinguished from each other based on the unweighted/weighted unifrac distance and relative abundance at the Phylum level (Figures 5C,D).

At the Phylum level, the relative abundances of Bacteroidetes and Verrucomicrobia were lower whereas the relative abundance of Firmicutes was higher in the $\mathrm{A}^{+} \mathrm{B}^{+}$subgroup when compared with the $\mathrm{A}^{-} \mathrm{B}^{+}$and $\mathrm{A}^{-} \mathrm{B}^{-}$subgroups (Figures 5C,D). At the Class level, the relative abundances of Bacteroidia and Verrucomicrobiae were lower and the relative abundances of Clostridia and Bacilli were higher in the $\mathrm{A}^{+} \mathrm{B}^{+}$subgroup (Figure 6A). At the Order level, the 
A

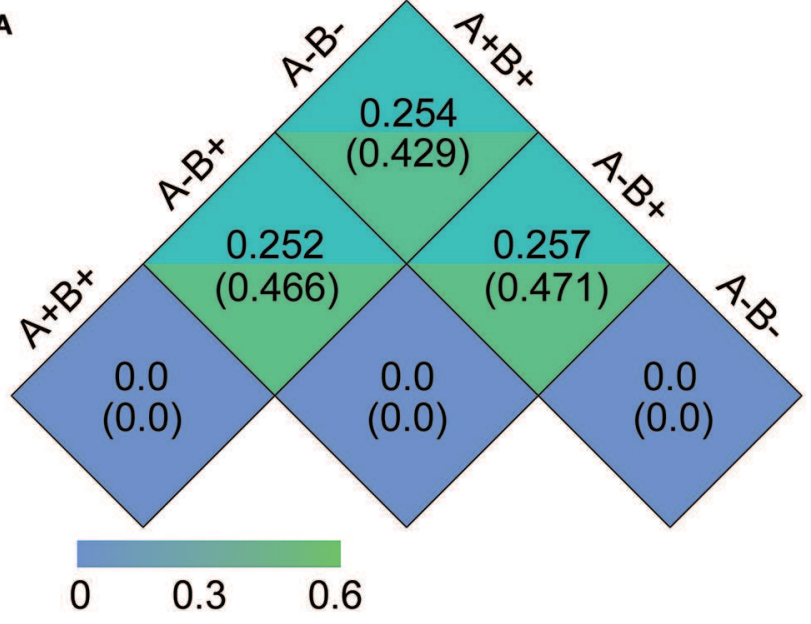

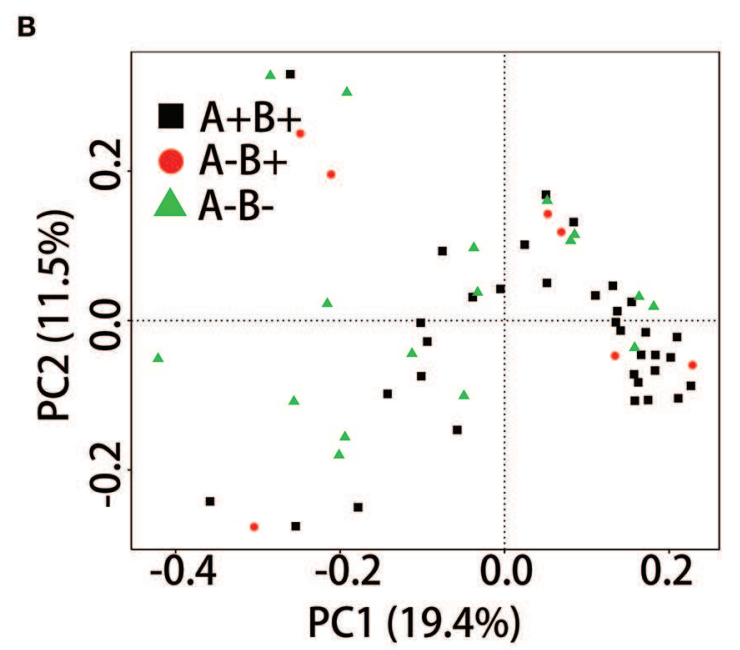

C

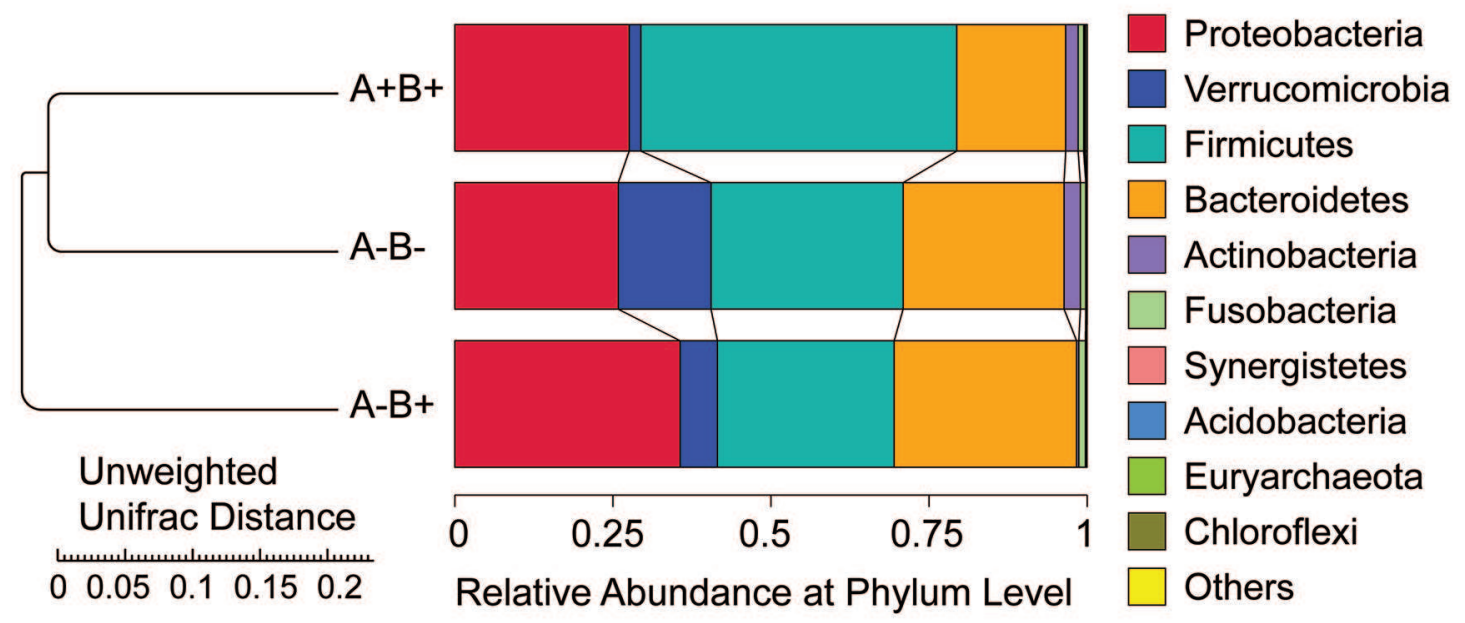

D

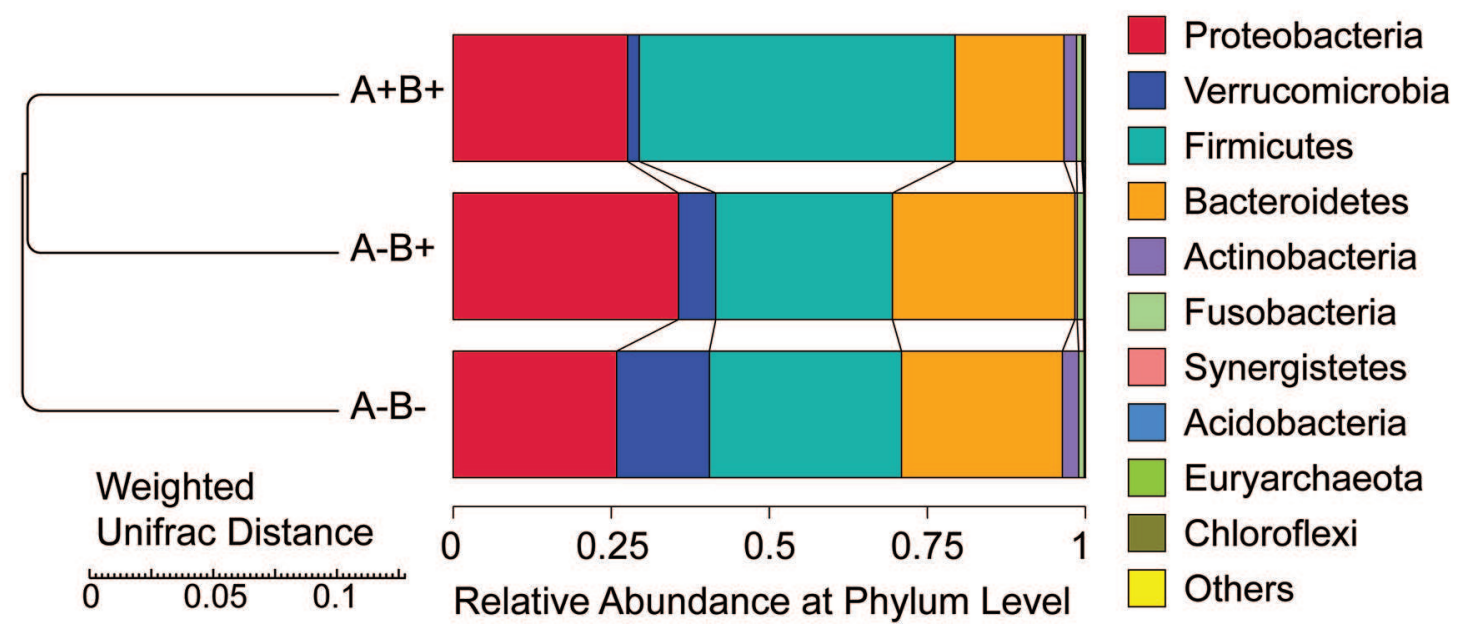

FIGURE 5 | $\alpha$-diversity and $\beta$-diversity analysis in subgroups 16 S rDNA V4 sequencing analysis. (A) Unifrac distance between groups. Upper number: weighted unifrac distance; lower number: un-weighted unifrac distance. Both numbers represent the index for differences in taxon-diversity between groups. (B) PCoA based on unweighted unifrac distance. (C) Sample clustering based on unweighted unifrac distance and relative abundance at Phylum level. (D) Sample clustering based on weighted unifrac distance and relative abundance at Phylum level. 


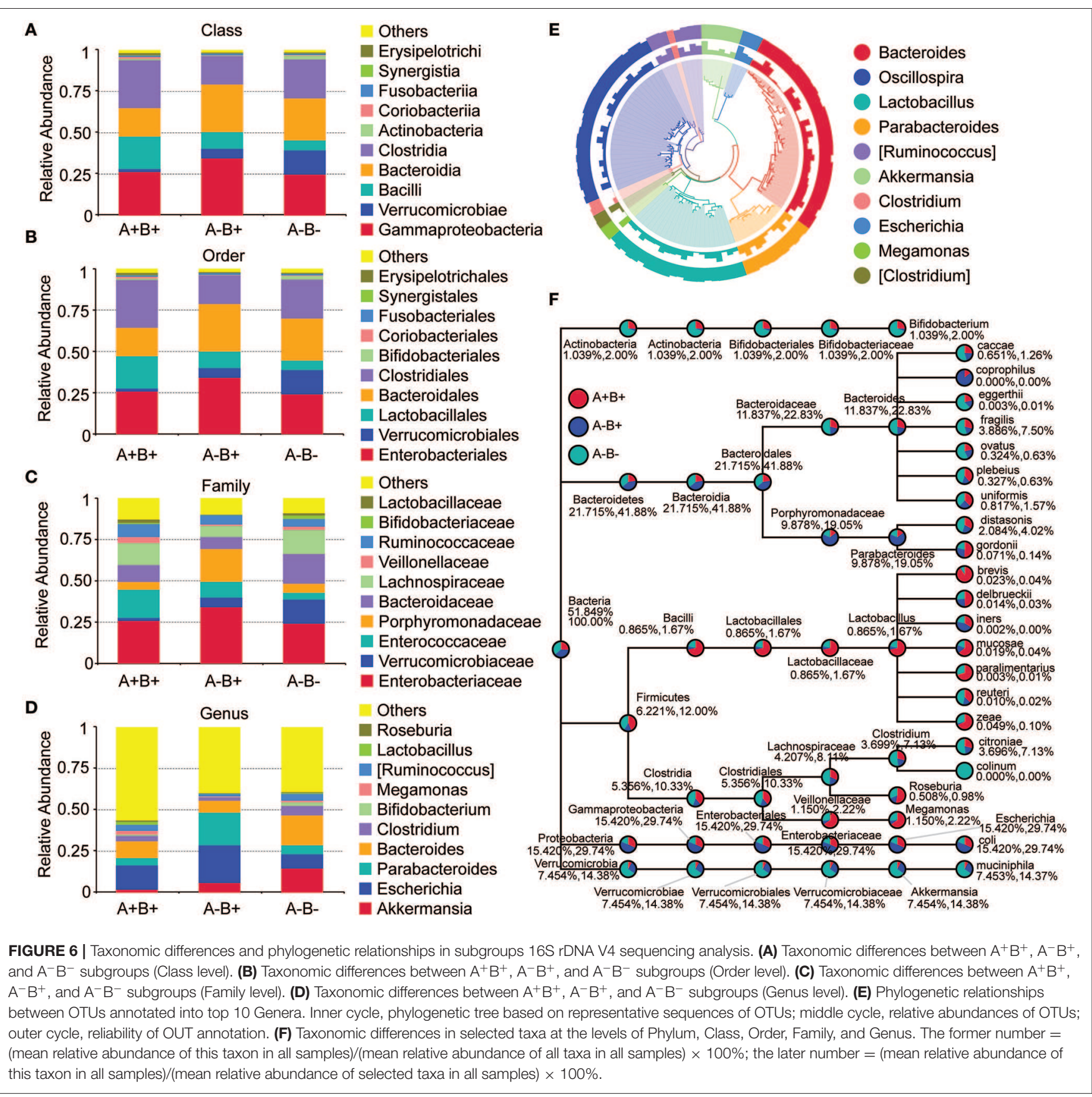

relative abundances of Bacteroidales and Verrucomicrobiales were lower and the relative abundances of Clostridiales and Lactobacillales were higher in the $\mathrm{A}^{+} \mathrm{B}^{+}$subgroup (Figure 6B). At the Family level, the relative abundance of Verrucomicrobiaceae was lower and the relative abundances of Enterococcaceae and Ruminococcaceae were higher in the $\mathrm{A}^{+} \mathrm{B}^{+}$subgroup (Figure $6 \mathrm{C}$ ). At the Genus level, the relative abundance of Akkermansia was lower in the $\mathrm{A}^{+} \mathrm{B}^{+}$subgroup (Figure 6D). Moreover, Methylococcaceae, Geobacteraceae, Peptococcaceae, Crenotrichaceae, Mogibacteriaceae, Acetobacter, Dialister, and Crenothrix significantly increased in the $\mathrm{A}^{+} \mathrm{B}^{+}$subgroup in comparison with the $\mathrm{A}^{-} \mathrm{B}^{-}$subgroup.
Exiguobacteraceae, Rickettsiaceae, Promicromonosporaceae, Procabacteriaceae, Providencia, Cellulosimicrobium, Wolbachia, and Saccharopolyspora significantly decreased in the $\mathrm{A}^{+} \mathrm{B}^{+}$ subgroup in comparison with the $\mathrm{A}^{-} \mathrm{B}^{-}$subgroup.

Phylogenetic relationships between the representative sequences of all OTUs corresponding to the top 10 taxa in Genus were studied (Figure 6E), and a taxonomic tree was constructed (Figure 6F). Bacteroides eggerthii, Bacteroides fragilis, Bacteroides ovatus, and Clostridium citroniae were more abundant in the $\mathrm{A}^{-} \mathrm{B}^{-}$subgroup than in the $\mathrm{A}^{-} \mathrm{B}^{+}$ and $\mathrm{A}^{+} \mathrm{B}^{+}$subgroups. These taxa might be suppressed by the presence of toxins. The abundances of Proteobacteria, 
Gammaproteobacteria, Enterobacteriales, Enterobacteriaceae, Escherichia, and Escherichia coli in the $\mathrm{A}^{-} \mathrm{B}^{-}$subgroup $\mathrm{A}^{-} \mathrm{B}^{+}$ subgroup were much lower than in the $\mathrm{A}^{+} \mathrm{B}^{+}$and $\mathrm{A}^{-} \mathrm{B}^{-}$ subgroups. These taxa might be associated with $\mathrm{A}^{-} \mathrm{B}^{+}$.

\section{DISCUSSION}

To better understand how the microbiome is associated with diarrhea, $\mathrm{CDpD}$, and $\mathrm{C}$. difficile type, we characterized the gut microbiota of healthy individuals and ICU individuals with $\mathrm{CDpD}$ or $\mathrm{CDnD}$. Consequently, we found gut microbiota compositions with potential associations with $\mathrm{CDpD}$ and $C$. difficile type in ICU patients.

Analyses of the Shannon index and number of observed species indicated that the microbial diversity of the $\mathrm{CDpD}$ group was lower than that of the $\mathrm{CDnD}$ and control groups, and this agreed well with a previous study in elderly (age $\geq$ 65) hospitalized patients (Milani et al., 2016). The unweighted unifrac distance between $\mathrm{CDpD}$ and control/CDnD was higher than that between $\mathrm{CDnD}$ and control. In PCoA, CDpD samples could be distinguished from $\mathrm{CDnD}$ samples and controls, whereas $\mathrm{CDnD}$ samples could not be separated from controls. These results indicated that the differences in taxon diversity between $\mathrm{CDpD}$ and control/CDnD were much higher than those between $\mathrm{CDnD}$ and control and that the appearance of $C$. difficile was strongly associated with the decrease in diversity of the gut microbiota.

Differences in community composition between groups were investigated. Metagenome analysis revealed that the abundances of 10 taxa (e.g., Deferribacteres, Cryptomycota, Acetothermia) in the $\mathrm{CDpD}$ group were significantly higher than in the $\mathrm{CDnD}$ group. Additionally, the abundances of two taxa (Saccharomycetes and Clostridia) were significantly lower in $\mathrm{CDpD}$ in comparison with control. Saccharomyces boulardii is a nonpathogenic yeast that promotes intestinal immunoglobulin-A immune response to $C$. difficile toxin $\mathrm{A}$ and thus protects against recurrent $C$. difficile colitis and diarrhea (Qamar et al., 2001). A balanced gut microbiota is characterized by conserved features like the predominance of Bacteroidia and Clostridia (Winter and Baumler, 2014). Therefore, $\mathrm{CDpD}$ might be associated with a decrease in beneficial taxa (i.e., Saccharomycetes and Clostridia) and an increase in harmful taxa (e.g., Deferribacteres, Cryptomycota, Acetothermia) in gut microbiota. Also, Saccharomycetes and Clostridia might serve as future candidate targets in ICU CDpD patients to help them re-establish healthy gut microbiota. Saccharomyces boulardii has been utilized to prevent antibioticassociated diarrhea in adult hospitalized patients in clinical trials (Ehrhardt et al., 2016).

Moreover, based on $16 \mathrm{~S}$ rDNA sequencing data, relative abundances of some taxa (e.g., Gammaproteobacteria, Enterobacteriaceae, Porphyromonadaceae, and Akkermansia) were higher in the $\mathrm{CDpD}$ group in comparison with the $\mathrm{CDnD}$ and control groups. Our results were partially consistent with previous study, in which Gammaproteobacteria and
Enterobacteriaceae were significantly over-represented in CDI samples, whereas Porphyromonadaceae and Akkermansia were significantly under-represented in CDI samples, in comparison with non-CDI samples. Reportedly, A. muciniphila is an intestinal representative of the Verrucomicrobia, and it associates with health in humans. Reduction of $A$. muciniphila was observed in patients with inflammatory bowel diseases, which led to the hypothesis that A. muciniphila possesses health-promoting activities and anti-inflammatory properties (Derrien et al., 2016). Although Akkermansia may improve barrier function, its over-representation in $\mathrm{CDpD}$ may be caused by modifications in the gut micro-environment and reflects enteric mucosa inflammation (Zwielehner et al., 2011). Thus, CDpD might also correlate with increase in Akkermansia.

Furthermore, differences in microbiota pathways between the three groups were studied using metagenome sequencing data. "Lysine biosynthesis" and "Coenzyme B biosynthesis" were only annotated in $\mathrm{CDpD}$ samples. Therefore, $\mathrm{CDpD}$ might be related with these pathways in gut microbiota, and drugs inhibiting these pathways or the colonization of Deferribacteres, Cryptomycota, Acetothermia, Gammaproteobacteria, and Enterobacteriaceae might be effective in limiting $C$. difficile blooming.

In the $\mathrm{CDpD}$ group, the community richness and microbial diversity of the $\mathrm{A}^{+} \mathrm{B}^{+}$subgroup were higher than those of the $\mathrm{A}^{-} \mathrm{B}^{+}$and $\mathrm{A}^{-} \mathrm{B}^{-}$subgroups. The similar unweighted unifrac distances (or weighted unifrac distances) between $\mathrm{A}^{-} \mathrm{B}^{+}$and $\mathrm{A}^{-} \mathrm{B}^{-}$, between $\mathrm{A}^{+} \mathrm{B}^{+}$and $\mathrm{A}^{-} \mathrm{B}^{-}$, and between $\mathrm{A}^{+} \mathrm{B}^{+}$and $\mathrm{A}^{-} \mathrm{B}^{+}$indicated similar differences in taxon diversity between these subgroups. High inter-sample variability was found in PCoA, and $\mathrm{A}^{+} \mathrm{B}^{+}$samples could not be distinguished from $\mathrm{A}^{-} \mathrm{B}^{+}$or $\mathrm{A}^{-} \mathrm{B}^{-}$samples. Besides, some taxa were significantly different between the $\mathrm{A}^{+} \mathrm{B}^{+}$ and $\mathrm{A}^{-} \mathrm{B}^{-}$subgroups (e.g., Acetobacter, Saccharopolyspora). These results suggested that higher sample numbers in the $\mathrm{A}^{+} \mathrm{B}^{+}, \mathrm{A}^{-} \mathrm{B}^{+}$, and $\mathrm{A}^{-} \mathrm{B}^{-}$subgroups are required for conclusive results.

Unfortunately, some limitations were found in this study. Firstly, we did not collect the reasons for diarrhea in the CDnD group. Secondly, age and antibiotic usage were not matched among the three groups, as the clinical complexity of ICU patients and the application of polypharmacy did not allow us to identify a sufficient number of subjects for each group or to standardize antibiotic treatment. In our future studies, more subjects will be enrolled to obtain a better stratification of patient features at baseline, and more clinical data (e.g., the reasons for diarrhea in the $\mathrm{CDnD}$ group) will be collected.

\section{CONCLUSIONS}

In ICU patients, the development of $\mathrm{CDpD}$ might be associated with alterations in gut microbiota, including decreases in beneficial taxa (i.e., Saccharomycetes and Clostridia) and increases in Deferribacteres, Cryptomycota, Acetothermia, Gammaproteobacteria, and Enterobacteriaceae. Besides, C. 
difficile toxin types might slightly influence gut microbiota composition in ICU patients with CDpD. These results provided a further understanding of the mechanism of $\mathrm{CDpD}$ in ICU patients and shed light on future directions for developing novel therapies to re-construct healthy gut microbiota.

\section{DATA AVAILABILITY STATEMENT}

The data supporting the conclusion of this article were submitted to SRA NCBI; the SRA accession information is PRJNA597086 (16s rDNA) and PRJNA591064 (metagenome).

\section{ETHICS STATEMENT}

This study was approved by the Ethics Committee of Xiangya Hospital Central South University, China (ID 201212027). All participants granted written informed consent before enrollment, and all investigations followed the principles of the Declaration of Helsinki.

\section{AUTHOR CONTRIBUTIONS}

AW, JD, and CL conceived and designed the experiments. JD, SL, $\mathrm{CZ}$, and XM performed the experiments. CF, PZ, QD, and CL

\section{REFERENCES}

Bagdasarian, N., Rao, K., and Malani, P. N. (2015). Diagnosis and treatment of Clostridium difficile in adults: a systematic review. JAMA 313, 398-408. doi: 10.1001/jama.2014.17103

Britton, R. A., and Young, V. B. (2012). Interaction between the intestinal microbiota and host in Clostridium difficile colonization resistance. Trends Microbiol. 20, 313-319. doi: 10.1016/j.tim.2012. 04.001

Centers for Disease Control and Prevention (CDC) (2012). Vital signs: preventing Clostridium difficile infections. MMWR Morb. Mortal Wkly. Rep. 61, 157-162.

Derrien, M., Belzer, C., and De Vos, W. M. (2016). Akkermansia muciniphila and its role in regulating host functions. Microb. Pathog. 106, 171-181. doi: 10.1016/j.micpath.2016.02.005

Ehrhardt, S., Guo, N., Hinz, R., Schoppen, S., May, J., Reiser, M., et al. (2016). Saccharomyces boulardii to prevent antibiotic-associated diarrhea: a randomized, double-masked, placebo-controlled trial. Open Forum. Infect. Dis. 3:ofw011. doi: 10.1093/ofid/ofw011

Kato, H., Kato, N., Watanabe, K., Iwai, N., Nakamura, H., Yamamoto, T., et al. (1998). Identification of toxin A-negative, toxin B-positive Clostridium difficile by PCR. J. Clin. Microbiol. 36, 2178-2182.

Kelly, C. R., Kahn, S., Kashyap, P., Laine, L., Rubin, D., Atreja, A., et al. (2015). Update on fecal microbiota transplantation 2015: indications, methodologies, mechanisms, and outlook. Gastroenterology 149, 223-237. doi: 10.1053/j.gastro.2015.05.008

Lee, J.-C., Hung, Y.-P., Tsai, P.-J., and Ko, W.-C. (2015). Clostridium difficile-associated diarrhea in medical intensive care units of a medical center in southern Taiwan: a prospective surveillance study. J. Microbiol. Immunol. Infect. 48:S182. doi: 10.1016/j.jmii.2015. 02.637

Leffler, D. A., and Lamont, J. T. (2015). Clostridium difficile infection. N. Engl. J. Med. 373, 287-288. doi: 10.1056/NEJMc1506004

Lemee, L., Dhalluin, A., Testelin, S., Mattrat, M. A., Maillard, K., Lemeland, J. F., et al. (2004). Multiplex PCR targeting tpi (triose phosphate isomerase), tcdA (Toxin A), and $\operatorname{tcdB}$ (Toxin B) genes for toxigenic analyzed the data. CL and AW wrote the paper. All authors read and approved the final manuscript.

\section{FUNDING}

This work was supported by the National Natural Science Foundation of China (No. 81601803), the Research Fund of Hunan Provincial Health and Family Planning Commission (No. B2016107), Young Scientists Fund of Xiangya Hospital (2014Q05), and Xiangya Sinobio Way Health Research Fund (No. xywm2015I11). The funders had no role in study design, data collection and analysis, decision to publish, or preparation of the manuscript.

\section{ACKNOWLEDGMENTS}

This manuscript has been released as a Pre-Print at bioRxiv.

\section{SUPPLEMENTARY MATERIAL}

The Supplementary Material for this article can be found online at: https://www.frontiersin.org/articles/10.3389/fcimb. 2020.00190/full\#supplementary-material culture of Clostridium difficile. J. Clin. Microbiol. 42, 5710-5714. doi: 10.1128/JCM.42.12.5710-5714.2004

Li, C., Li, Y., Huai, Y., Liu, S., Meng, X., Duan, J., et al. (2018). Incidence and outbreak of healthcare-onset healthcare-associated clostridioides difficile infections among intensive care patients in a large teaching hospital in China. Front. Microbiol. 9:566. doi: 10.3389/fmicb.2018. 00566

Milani, C., Ticinesi, A., Gerritsen, J., Nouvenne, A., Lugli, G. A., Mancabelli, L., et al. (2016). Gut microbiota composition and Clostridium difficile infection in hospitalized elderly individuals: a metagenomic study. Sci. Rep. 6:25945. doi: $10.1038 /$ srep 25945

Persson, S., Jensen, J. N., and Olsen, K. E. (2011). Multiplex PCR method for detection of Clostridium difficile tcdA, tcdB, cdtA, and $\operatorname{cdtB}$ and internal in-frame deletion of tcdC. J. Clin. Microbiol. 49, 4299-4300. doi: 10.1128/JCM.05161-11

Qamar, A., Aboudola, S., Warny, M., Michetti, P., Pothoulakis, C., Lamont, J. T., et al. (2001). Saccharomyces boulardii stimulates intestinal immunoglobulin a immune response to Clostridium difficile toxin a in mice. Infect. Immun. 69, 2762-2765. doi: 10.1128/iai.69.4.2762-276 5.2001

Rupnik, M., Wilcox, M. H., and Gerding, D. N. (2009). Clostridium difficile infection: new developments in epidemiology and pathogenesis. Nat. Rev. Microbiol. 7, 526-536. doi: 10.1038/nrmicr o2164

Schubert, A., Rogers, M., Ring, C., Mogle, J., Petrosino, J., Young, V., et al. (2014). Microbiome data distinguish patients with Clostridium difficile infection and non-C. difficile-associated diarrhea from healthy controls. mBio 5, e01021e01014. doi: 10.1128/mBio.01021-14

Shivashankar, R., Khanna, S., Kammer, P. P., Harmsen, W. S., Zinsmeister, A. R., Baddour, L. M., et al. (2013). Clinical factors associated with development of severe-complicated Clostridium difficile infection. Clin. Gastroenterol. Hepatol. 11, 1466-1471. doi: 10.1016/j.cgh.2013.04.050

Stevens, V., Dumyati, G., Fine, L. S., Fisher, S. G., and Van Wijngaarden, E. (2011) Cumulative antibiotic exposures over time and the risk of Clostridium difficile infection. Clin. Infect. Dis. 53, 42-48. doi: 10.1093/cid/cir301 
Vincent, J. L., Bassetti, M., Francois, B., Karam, G., Chastre, J., Torres, A., et al. (2016). Advances in antibiotic therapy in the critically ill. Crit. Care 20:133. doi: 10.1186/s13054-016-1285-6

Winter, S. E., and Baumler, A. J. (2014). Dysbiosis in the inflamed intestine: chance favors the prepared microbe. Gut. Microbes 5, 71-73. doi: 10.4161/gmic.27129

Yassin, S. F., Young-Fadok, T. M., Zein, N. N., and Pardi, D. S. (2001). Clostridium difficile-associated diarrhea and colitis. Mayo Clin. Proc. 76, 725-730. doi: 10.4065/76.7.725

Zwielehner, J., Lassl, C., Hippe, B., Pointner, A., Switzeny, O. J., Remely, M., et al. (2011). Changes in human fecal microbiota due to chemotherapy analyzed by TaqMan-PCR, 454 sequencing and PCR-DGGE fingerprinting. PLoS ONE 6:e28654. doi: 10.1371/journal.pone.0028654
Conflict of Interest: The authors declare that the research was conducted in the absence of any commercial or financial relationships that could be construed as a potential conflict of interest.

Copyright (c) 2020 Duan, Meng, Liu, Zhou, Zeng, Fu, Dou, Wu and Li. This is an open-access article distributed under the terms of the Creative Commons Attribution License (CC BY). The use, distribution or reproduction in other forums is permitted, provided the original author(s) and the copyright owner(s) are credited and that the original publication in this journal is cited, in accordance with accepted academic practice. No use, distribution or reproduction is permitted which does not comply with these terms. 